Para enlazar con este artículo / To link to this article:

http://dx.doi.org/10.14198/fem.2018.31.02

Para citar este artículo / To cite this article:

Del Barrio Álvarez, Elena. «Satisfacción revolucionaria. Un modelo de intervención psicológica para supervivientes de trata que hacen trabajo sexual después de los 40». En Feminismo/s, 31 (junio 2018): 39-63. Dosier monográfico: Sexo y bienestar. Mujeres y diversidad, coords. Carmen Mañas Viejo y Alicia Martínez Sanz, DOI: 10.14198/fem.2018.31.02

\title{
SATISFACCIÓN REVOLUCIONARIA. UN MODELO DE INTERVENCIÓN PSICOLÓGICA PARA SUPERVIVIENTES DE TRATA QUE HACEN TRABAJO SEXUAL DESPUÉS DE LOS 40
}

\author{
REVOLUTIONARY SATISFACTION. A PSYCOLOGICAL \\ INTERVENTION MODEL FOR TRAFFICKING SURVIVORS \\ WHO DO SEX WORK AFTER THEIR 40'S
}

\author{
Elena DEL BARRIO ÁLVAREZ \\ Investigadora en el Consejo Superior de Investigaciones ciéntificas (CSIC) \\ orcid.org/0000-0003-1686-9539
}

\section{Resumen}

Las medidas existentes para fomentar el restablecimiento de supervivientes de trata sexual deben mejorar su inclusión. Mujeres supervivientes que realizan trabajo sexual corren el riesgo de quedar excluidas, situación agravada con su envejecimiento, y con las etiquetas patológicas que reciben. La presente investigación toma como protagonistas a dos grupos de mujeres supervivientes de trata que realizan trabajo sexual, uno de 30 menores de 26 años, y otro de 30 mujeres mayores de 40 años. Con ellas se explora la adecuación de un modelo extraído de la Teoría Socio Cognitiva de Bandura (1997). Los resultados del análisis de moderación realizados con SPSS v23 y la macro de PROCESS, indican que los incrementos en los niveles de autoeficacia solo parecen predictores de aumentos en la satisfacción con la vida en el grupo de mayor edad. Por lo tanto, podría ser pertinente realizar intervenciones diferenciales en función de su edad. (CC BY 4.0)

Feminismo/s 31, junio 2018, pp. 39-63 
Palabras clave: prostitución, trata con fines de explotación sexual, satisfacción con la vida, autoeficacia, envejecimiento.

\begin{abstract}
The existing measures to encourage the re-establishment of sex trafficking survivors should be improved. Trafficking survivors who perform sex work are in risk of being excluded, a situation aggravated by their aging and their psychological pathologization. Therefore, the present investigation takes as protagonists two groups of women trafficking survivors who perform sex work. One of 30 adults under 26, and another group of 30 over 40 years old. With them, it is explored the adequacy of a psychological intervention model extracted from the Socio-Cognitive Theory of Bandura (1997). The results of the moderation analysis performed with SPSS v23 and the PROCESS macro indicate that increases in self-efficacy levels only seem to predict increases in life satisfaction in the older age group. Therefore, it may be pertinent to carry out differential interventions according to their age.
\end{abstract}

Keywords: Prostitution, Trafficking for sexual exploitation, Satisfaction with life, Self-efficacy, Aging.

\title{
1. INTRODUCCIÓN
}

La violencia machista ocupa las horas de las mujeres. Somos agredidas en la calle, en el hogar, en la cama, en el trabajo... El acoso callejero, las violaciones por parte de nuestras parejas, los techos de cristal, los artículos pseudo-científicos sobre la ideología de género, la tolerancia mediática de las agresiones sexuales, las disculpas sociales existentes que protegen a nuestros agresores, las dificultades para denunciar y acabar con la violencia sexual..., son parte de la cultura patriarcal en la que todas sobrevivimos. Nuestros cuerpos son campos de batalla, y una exploración de lo que nos provoca cada parte de nuestro cuerpo, nos puede dar pistas sobre cómo nos han agredido (Aldama 60). Por ejemplo, nuestro pudor sexual nos puede mostrar la violencia y represión sexual a las que tenemos que sobrevivir. De hecho, la violencia de género y la violencia sexual están estrechamente ligadas, sobre todo cuando incluimos interseccionalidades de opresiones (Bay-Cheng y Bruns 504). Es decir, no todas tenemos las mismas facilidades ni el mismo apoyo para lidiar con las violencias machistas. Factores como nuestra clase social, identidad 
sexual, edad, o nacionalidad, marcarán las posibilidades que se nos ofertarán para reducir el grado de violencia que recibimos. El incremento del número de opresiones que nos constriñan, incrementará las posibilidades de que las violencias machistas que recibimos, sean en mayor grado explicitadas sexualmente. Esto sucede porque se produce una erotización de lo sometido, por lo que, a mayor sometimiento, mayor erotización (Armstrong; Hamilton y Armstrong 100).

Por ello, no es casual que la explotación sexual sea ejercida sobre todo en mujeres, pobres, migrantes e ilegales. Frecuentemente son noticia las agresiones sexuales que sufrimos mujeres blancas, universitarias, jóvenes, de clase media-alta... Sin embargo, pese a la elevada incidencia, no son noticia las agresiones dirigidas a prostitutas, pobres, migrantes, ilegales, viejas... (Jackson 46). Ninguna mujer tiene fácil la convivencia con las violencias machistas que le agreden, pero debemos darnos cuenta de que nuestras heridas no son las únicas, ni las más profundas. Por lo tanto, para que dejen de existir las violencias machistas, debemos eliminarlas de todos los contextos, y no sólo del que tenemos más próximo.

En el presente trabajo se observa un contexto particular, del que se presume existe poca labor realizada desde el punto de vista de la psicología clínica: las supervivientes de trata con fines de explotación sexual que se dedican al trabajo sexual. Es decir, de aquellas mujeres que fueron víctimas de un crimen de explotación sexual (prostitución ejercida en términos no consentidos), y que una vez sobrevivido a dicha experiencia (cese de la relación de explotación), deciden ejercer la prostitución en las calles de Madrid (de forma voluntaria).

Se considera que las supervivientes de trata sexual son supervivientes de un tipo de violencia de género, dado que la mayoría de las víctimas son mujeres, y cuya incidencia del crimen es facilitada por el sistema patriarcal que nos estructura (Merry 75). Es decir, la alianza entre patriarcado y capital es el germen que favorece el caldo de cultivo de violencias laborales, sexuales y afectivas que promueven la feminización de la pobreza y la explotación de los cuerpos femeninos (Cobo 12). O lo que es lo mismo, la tolerancia a la violencia sexual hacia las mujeres, unida al monstruo de explotación económica que supone el capitalismo, provocan que la forma de trata de seres humanos

Feminismo/s 31, junio 2018, pp. 39-63 
más frecuente sea la trata de mujeres con fines de explotación sexual (EU, Comission 4).

La erradicación de este tipo de trata es uno de los objetivos marcados por la Unión Europea, y entre las medidas que plantea para su consecución está el favorecer el restablecimiento de las supervivientes a dicho crimen. Por ello, se subvencionan organizaciones que ofrecen asilo y asistencia psicosocial a estas mujeres. Sin embargo, las bases y condiciones en las que se ofrece esta asistencia no permiten la inclusión de todas las mujeres supervivientes (Pérez Alonso 713). Este es el caso de las mujeres que después de la experiencia de trata sexual realizan trabajo sexual. La actual legislación ofrece amparo exclusivamente a aquellas mujeres que, habiendo sido detectadas como víctimas de trata, hayan cortado cualquier nexo con sus redes de explotación, lo cual se operativiza en la negación de asistencia a quienes realicen trabajo sexual (Ministerio de Sanidad, Servicios Sociales e Igualdad 23).

De este modo, las asociaciones que ofrecen atención psicológica a supervivientes de trata, no contemplan una atención específica para aquellas que ejerzan prostitución, ni tampoco se conocen publicaciones que propongan un marco de intervención adecuado a su situación. Asimismo, una vez cumplida la mayoría de edad, no existen asociaciones dedicadas a etapas del ciclo vital específicas, y los recursos que se ofrecen son generales para todas las mujeres (Ministerio de Sanidad, Servicios Sociales e Igualdad 17).

No obstante, una de las principales salidas laborales después de la trata sexual es el ejercicio de la prostitución. Negar la existencia de esta decisión, y despistar los deberes de recompensa que la sociedad tiene ante ellas, supone un atentado en contra de sus derechos humanos. Asimismo, en el caso de las mujeres mayores de 40 años en esta situación, la complejidad aumenta (Freixas et al. 37). En el caso de las nacionales, se enfrentan a un mercado laboral que las excluye, en el caso de migrantes, es probable que ni se les otorgue el permiso de trabajo. Por otra parte, pese a haber sido explotadas en beneficio de la sociedad, no se les reconoce jubilación, en tanto que no han cotizado. Estas condiciones provocan que la decisión de percibir ingresos económicos por medio de la prostitución sea el medio más adecuado ante su situación. Sin embargo, en las investigaciones y en los planes de intervención permanecen invisibilizadas, como si ésta no fuera la opción más frecuente.

Feminismo/s 31, junio 2018, pp. 39-63 
Esta decisión no es tomada en cuenta, porque no se considera pertinente ofertar una asistencia diferencial. El contexto de alegalidad, de carencia de derechos y el peso del estigma social, pueden provocar unas necesidades específicas no cubiertas por modelos de intervención generales. De todos modos, con independencia de si requieren o no un tipo de asistencia diferente, se considera necesario visibilizar su existencia y detenernos a reflexionar sobre el contexto psicosocial que las engloba. Por ello, se toma a estas mujeres como protagonistas de esta investigación. Además, debido a la carencia de recursos específicos para mayores de 40 años dentro de este colectivo, se considera idóneo observar si efectivamente ese tratamiento genérico de la edad es adecuado.

A pesar de reconocer la necesidad de proponer marcos de intervención que se centren en reducir las posibles secuelas más visiblemente desagradables (ansiedad, depresión, trastorno de estrés postraumático), para evitar el encasillamiento que se produce en la triada mujer-víctima-patología, en la presente investigación se pone el foco en la necesidad de observar e incrementar las posibles fortalezas con las que se puede trabajar a nivel psicológico. Romper con las investigaciones patologizantes y promover el bienestar de las mujeres son objetivos de la psicología feminista (Worrel 336). Además, el bienestar es algo más que la ausencia de malestar (Seligman 559), y por ello la detección e incremento de fortalezas son objetivos de la psicología clínica.

De este modo, la presente investigación propone un modelo para potenciar el bienestar psicológico de las mujeres protagonistas de esta investigación, concretamente, su satisfacción con la vida. El modelo está basado en la Teoría Cognitivo Social de Bandura (1997), y es testado en supervivientes adultas menores de 26 años y mayores de 40 años. Los resultados aportan información para mejorar el sistema de atención psicológica que actualmente se les ofrece.

¿Por qué es interesante incrementar la satisfacción con la vida en esta población?

En la actual sociedad del bienestar, la expresión de emociones desagradables, o el reconocimiento de enfermedades transitorias son signos de debilidad (Lai 43). Esta debilidad mengua el capital simbólico de una persona. La insatisfacción se asocia a fracaso, y el fracaso a la exclusión. Por ello, la psicología positiva, y las intervenciones sobre la satisfacción con la vida en la población general, están triunfando y extendiéndose vertiginosamente (Bannink 216). 
Sin embargo, cuando nos centramos en violencias ejercidas específicamente sobre mujeres, sobre nuestros cuerpos y nuestras sexualidades, los enfoques están más centrados en lo patológico que en volver a generar experiencias de bienestar (Brunovskis 53). Por supuesto, el bienestar hedónico parece aún más inaccesible a nivel clínico para sectores poblacionales marginalizados (McKenzie et al. 136). Pese a reconocer la importancia del trabajo centrado en el trauma, se considera que este no ha de ser el único, y menos ha de serlo exclusivamente en poblaciones apartadas de la centralidad de las políticas de bienestar, puesto que esto agrava su situación. Las supervivientes de trata sexual que ejercen prostitución, son evidentemente unas de las afectadas. Por ello, en esta ocasión se considera oportuno poner el foco en su satisfacción con la vida. Con los resultados se pretende plasmar cómo la existencia transitoria o localizada de determinados deterioros no significa que no existan potencialidades ni virtudes que puedan incentivarse dentro del ámbito clínico (Vázquez y Pérez-Sales 231).

Además, se considera conveniente incluir la satisfacción con la vida, no sólo por los beneficios que reporta en términos de capital simbólico, también por su potencial para disminuir la alienación y para incrementar la participación social (Goodwin, Cook y Young 225). Estos resultados son cruciales en un sector poblacional cuya elección laboral es negada legislativamente y, por lo tanto, su representatividad en las políticas públicas es nula, marginal o casual (Holgado 30). Incrementar una variable que potencia las probabilidades de participación social podría ser fundamental para propiciar su restablecimiento. Además, la reducción de la alienación facilita la generación de colectivos empoderados que luchan por sus derechos (Losier et al. 153), algo altamente recomendable en este sector poblacional teniendo en cuenta la falta de amparo legal y social con el que cuentan en la actualidad.

Asimismo, a nivel clínico, la satisfacción con la vida está vinculada a una mejor salud mental y física, y a una mejora en la amortiguación de los factores estresantes a los que nos exponemos (Bernis 9). Esto provoca que sea una variable deseada para cualquier tipo de intervención, pero especialmente si consideramos que las personas con las que trabajamos están sometidas a factores estresantes antecedentes de daños psicológicos significativos, como es el caso del contexto que rodea a las supervivientes de las que aquí se habla. 
Al incluir la variable satisfacción con la vida como variable del estudio, se desea visibilizar la posibilidad de experimentar bienestar después de experiencias de violencia asociadas a la trata y al ejercicio de la prostitución callejera en la actualidad. Que el patriarcado declare la guerra a nuestros cuerpos no significa que haya acabado con nuestras fortalezas.

¿Por qué es interesante analizar el rol de la autoeficacia para promover la satisfacción con la vida en este colectivo?

La autoeficacia es la energía que habilita para enfrentar retos, permite enfrentarse a la adversidad y recuperarse de ella, es un componente central de la resiliencia humana (Connor y Davidson 76). Según la Teoría Cognitivo Social de Bandura, la autoeficacia es propulsora de la experiencia de satisfacción con la vida. A raíz de esta teoría, diversas investigaciones han testado los efectos de la autoeficacia sobre la satisfacción con la vida, obteniendo resultados congruentes con las investigaciones de Bandura.

De este modo, parece que en población general los incrementos de la autoeficacia dan lugar a incrementos en la satisfacción con la vida. Las explicaciones a este fenómeno van dirigidas sobre todo al vínculo entre la confianza en las propias capacidades para conseguir un objetivo, con el mantenimiento de la acción hasta su consecución. Esto provoca, o la consecución de los objetivos, o la esperanza de conseguirlos, y, por lo tanto, un resultado positivo tras el resto de objetivos conseguidos y los propuestos, o lo que es lo mismo, un mantenimiento óptimo de la satisfacción con la vida.

A pesar de ello, sólo se tiene conocimiento de este suceso en población general. Las supervivientes de trata sexual se han enfrentado a un proceso específico de desempoderamiento, realizado por sus tratantes para evitar cualquier intento de huida (Zimmerman 8). También, para reclamar los derechos que les corresponden como supervivientes, se han tenido que enfrentar a un sistema institucional patriarcal que con frecuencia contempla a las mujeres víctimas como culpables, o cuya burocracia es ajena a los procesos emocionales de las supervivientes de violencia sexual y machista (López Martín 60). Dentro de este sistema, se invisibiliza cualquier agencialidad de las supervivientes, por lo que su percepción sobre sus capacidades puede estar alterada (Acale Sánchez 291). Además, al haber escogido realizar trabajo sexual, tienen 
que cargar con un estigma que las excluye socialmente (Weitzer 1). De este modo, el rol que puede jugar la autoeficacia en la generación de satisfacción con la vida puede variar su centralidad en esta población específica. De hecho, hay investigaciones que muestran que en trabajadoras sexuales la conexión es más compleja que la observada en población general (Freixas et al. 15). Se desconoce si sucede lo mismo con supervivientes de trata sexual que ejercen prostitución.

No obstante, podría haberse escogido cualquier otra variable vinculada tradicionalmente con los incrementos en satisfacción con la vida, como el estrés percibido, la ansiedad o la depresión. La elección de una variable desvinculada de lo patológico no ha sido casual. Las supervivientes de trata sexual son esencialmente victimizadas, lo cual dificulta el reconocimiento de su agencialidad y el desarrollo de su propia resiliencia, asimismo, quienes encima se dedican al trabajo sexual son frecuentemente o invisibilizadas o etiquetadas de desviadas (Lentin y Titley 130). Por ello, se ha considerado necesario evidenciar que el bienestar puede obtenerse interviniendo directamente en la generación de fortalezas, por lo que clínicamente, podemos evitar el incremento de la visión patologizante de este colectivo, sin por ello desatender el fomento de su bienestar.

En resumen, se considera conveniente analizar la potencialidad de la autoeficacia para incrementar la satisfacción con la vida en una población de supervivientes de trata que ejercen prostitución.

¿Por qué resulta conveniente analizar los efectos de la edad en esta investigación? La inclusión de la edad como variable de análisis resulta siempre interesante cuando se trabaja con población femenina y que pueda alejarse de la edad reproductiva Chrisler (6), Bell (75) y Sontag (629) lo recomendaron al observar la existencia de un doble criterio en la percepción de la edad en función del género. Encontraron que las mujeres somos más discriminadas por el envejecimiento que los hombres.

Además, si las investigaciones se centran en la promoción del bienestar, la inclusión del rol de la edad parece incrementar aún más su interés. Investigaciones previas muestran que la edad modula las variables implicadas en esta investigación. Como se explica a continuación, mientras que 
en población general se ha encontrado que la percepción de autoeficacia se mantiene estable a lo largo del ciclo vital (como defienden Brandtstädter y Rothermund, Freund y Baltes, y Heckhausen, Wrosch, y Schulz), la satisfacción con la vida parece variar.

Esto es, nuestras capacidades varían a lo largo del ciclo vital, y, por lo tanto, es de esperar que también varíe la confianza que poseemos en nuestras propias capacidades. Sin embargo, mediante mecanismos de asimilación y acomodación, los niveles de autoeficacia tienden a mantenerse estables. Es decir, ajustaríamos nuestras metas y nuestras estrategias para mantener nuestra percepción de eficacia estable a lo largo del ciclo vital.

No obstante, parece que la comorbilidad de trastornos psicológicos y la experiencia de bienestar varían en función de la edad (Thakur et al. 48). Estos fenómenos han mostrado ser disparadores de variaciones en los niveles de satisfacción con la vida (Geronimi, Paterson y Woodruff-Borden 430), la cual parece que aumenta con la edad (Lucas y Donnellan 323). Stewart, Ostrove, y Helson (23), encuentran que las mujeres a los 40 se sienten más empoderadas y capacitadas para enfrentarse a los retos.

Asimismo, no sólo es interesante analizar la edad cuando se trabaja de forma independiente con autoeficacia o satisfacción con la vida. Estudios previos nos indican que este interés se incrementa cuando trabajamos con un modelo que conecta a ambas. Perkins y Zimmerman (569) observaron que, pese a la importancia longitudinal del rol de la autoeficacia, es en las etapas más avanzadas de la vida cuando cobra un especial protagonismo. Es decir, aunque el sentimiento de autoeficacia impacta en la satisfacción con la vida a lo largo del ciclo vital, la edad varía la intensidad de su impacto a lo largo de éste, en tanto que varían las competencias y el auto-concepto.

A pesar de lo mencionado, Pinquart (414) añade que las poblaciones no representativas podrían experimentar una curva diferente a la de la población general. De hecho, en trabajadoras sexuales mayores de 40 años, se ha encontrado que los niveles de satisfacción con la vida son inferiores a los de la población general. Factores como el incremento de sentimientos de culpa en trabajadoras sexuales mayores de 40 años (Law 64), podrían afectar también a las supervivientes de trata que colaboran en esta investigación. Por todo ello, se considera interesante proponer un modelo específico para estas 
supervivientes de trata, y que atienda a las diferencias individuales en función de la edad, puesto que es posible que existan.

\section{DISEÑO}

En esta investigación se pretende analizar la potencialidad de un modelo para incrementar la satisfacción con la vida en dos grupos de supervivientes de trata sexual que ejercen trabajo sexual: un grupo de adultas menor de 26 años y otro grupo de mayores de 42 años.

El modelo propuesto es recuperado de la Teoría Cognitivo Social de Bandura, publicado en 1977. Se espera que la autoeficacia actúe como antecedente de la satisfacción con la vida y que la edad modere estos efectos (Jung y Oh 30). El modelo puede encontrarse en la Figura 1.

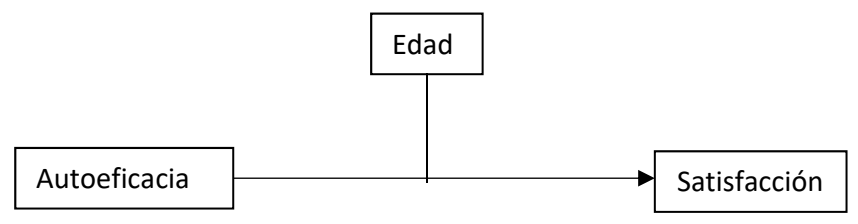

Figura 1. Modelo propuesto

\subsection{Las hipótesis que se ponen a prueba son:}

1. La autoeficacia será predictora de incrementos en la satisfacción con la vida.

2. La edad moderará el efecto de autoeficacia sobre satisfacción con la vida. Se espera que la satisfacción con la vida dependa en mayor grado de la autoeficacia en edades más avanzadas

\subsection{Método}

\section{Participantes}

En esta investigación colaboran 60 mujeres que fueron identificadas como víctimas de trata y que realizan trabajo sexual en las calles de Madrid. Ninguna de ellas comenzó el ejercicio de la prostitución antes de haber sido captadas por la red de trata sexual. Los datos se han recogido gracias a la colaboración 
con la Comisión de Investigación a Víctimas de Malos Tratos. 30 mujeres eran menores de 26 años y 30 mayores de 42 .

En el grupo de mujeres mayores de 42 años se encontró que 26 ejercían la prostitución en la zona centro y 4 en polígonos. En el de las mujeres menores de 26 años, 22 ejercían la prostitución en la zona centro y 8 en polígonos. En la Tabla 1 se puede apreciar en mayor detalle la información sociodemográfica recogida.

Tabla 1. Análisis descriptivo de variables sociodemográficas

\begin{tabular}{|l|l|c|c|c|c|}
\cline { 3 - 6 } \multicolumn{2}{c|}{} & \multicolumn{2}{c|}{ Menores de 26} & \multicolumn{2}{c|}{ Mayores de 42 } \\
\cline { 3 - 6 } \multicolumn{2}{c|}{ Cisgénero/Transgéro } & Total & $\%$ & \multicolumn{1}{c|}{ Total } & $\%$ \\
\hline \multirow{4}{*}{ Origen } & cis & 26 & 86.7 & 25 & 83.3 \\
\cline { 2 - 6 } & trans & 4 & 13.3 & 5 & 16.7 \\
\hline \multirow{3}{*}{ Estado civil } & Europa & 4 & 13.3 & 11 & 36.7 \\
\cline { 2 - 6 } & América Latina & 11 & 36.7 & 12 & 40.0 \\
\cline { 2 - 6 } & Africa & 15 & 50.0 & 7 & 23.3 \\
\hline Contacto con la familia & Soltera & 26 & 86.7 & 27 & 90 \\
\cline { 2 - 6 } & Con pareja & 3 & 10.0 & 3 & 10.0 \\
\cline { 2 - 6 } & Casada & 1 & 3.3 & 1 & 3.3 \\
\hline
\end{tabular}

\section{Procedimiento}

Previo al contacto con las participantes, 10 expertas revisaron los ítems del instrumento. Se pedía opinión sobre la pertinencia de las preguntas, el vocabulario y la longitud del instrumento final. Dichas apreciaciones fueron tenidas en cuenta en la elaboración del cuestionario. Además, debido a las características de la muestra (todas mujeres), el género de cada ítem se transformó al femenino.

Antes de facilitar el cuestionario se informaba de los fines de la investigación («conocer aspectos sobre su vida personal actual, no sobre la prostitución ni su pasado»), se les informaba de que rellenarían el cuestionario cerca de una encargada de resolver dudas, por si lo necesitaban, y que esta persona comprobaría que el cuestionario estuviera completado antes de proceder a 
la gratificación con dinero en efectivo. Una vez advertido y obtenido consentimiento oral, se pasaba un consentimiento informado por escrito en el que se indicaba que el tratamiento de los datos sería anónimo y con fines exclusivamente de investigación. Además, en éste también se indicaba que, al finalizar, su colaboración se recompensaría con 10 euros. La cantidad y pertinencia de dicha remuneración por la participación es recomendada por investigaciones que trabajan con supervivientes de trata (Zimmerman et al. 58), y por expertas en su restablecimiento psicológico. El cuestionario iba acompañado de una encuesta en la que se recogían variables sociodemográficas (ver Tabla 1).

\section{Medidas}

\section{Autoeficacia}

Se emplea la subescala ( 7 ítems) de autoeficacia del CD-RISC (construido para evaluar la resiliencia por Connor y Davidson). Se mantiene la escala tipo Liker de 5 puntos que va de $0=$ En absoluto a $4=$ Casi siempre. Un ejemplo de ítem es «No me desanimo fácilmente con el fracaso». Se elige esta versión reducida de la escala por el incremento de su consistencia interna con respecto a la versión extendida (Serrano-Parra 52). En el presente estudio se observa un alfa de Cronbach de 0.78

\section{Crecimiento Post-Traumático}

Se emplea la versión reducida de la escala PGTI de Tedeschi y Calhoun, The Posttraumatic Growth Inventory. Esta nueva estructura factorial del cuestionario está formada por 13 ítems que agrupan las dimensiones propuestas por los autores del cuestionario de: fuerza personal, relación con los demás y percepción de un cambio vital. Se emplea una escala tipo Likert de 6 puntos que va de $0=$ No cambio, a $5=$ Muy alto grado de cambio. Un ejemplo de ítem es «Tengo mayor apreciación por el valor de mi propia vida». En el presente estudio la escala presentó un alfa de 0.90 . 
Satisfacción con la vida

Se emplea la Escala de Satisfacción con la Vida (SWLS) de Diener, Emmons, Larsen y Griffin, en la versión que figura en el texto de Arce, Técnicas de construcción de escalas psicológicas. Está compuesta por 5 ítems y posee una escala tipo Likert de 5 puntos, donde $1=$ totalmente en desacuerdo, y $5=$ totalmente de acuerdo. Ejemplos de los ítems son «En la mayoría de los aspectos mi vida es como quiero que sea»; «Hasta ahora he conseguido de la vida las cosas que considero importantes». En el presente estudio la consistencia interna es de 0.83 .

\section{Edad}

Las mujeres incluidas en la investigación fueron seleccionadas en función de su edad. Se buscaron mujeres adultas menores de 26 años y mujeres mayores de 40 años. Fueron incluidas 30 mujeres menores de 26 y 30 mujeres mayores de 42 años. La mujer más joven tenía 18 años y la mayor, 68 años. Se escogen dichas franjas de edad por marcar franjas críticas en la experimentación de satisfacción con la vida en trabajadoras sexuales (Freixas et al. 7).

\section{Análisis}

Se emplea el SPSS versión 23 para el procesamiento de datos y la macro de PROCESS (de Preacher y Hayes 717) para el análisis de moderación. En un comienzo se procede a realizar un análisis descriptivo de las variables objeto de estudio. Se realiza la prueba $t$ de Student para analizar si hay diferencias en función de la edad con respecto a la satisfacción con la vida. Para minimizar los efectos de la multicolinealidad se realizaron todos los análisis de regresión con la variable independiente estandarizada (Aiken, West y Reno, Multiple regression). Para asegurar la validez del procedimiento, se analizaron los supuestos del modelo estadístico de normalidad, homocedasticidad y no colinealidad. Una vez efectuados los análisis, el diagrama de dispersión de los pronósticos por los residuos tipificados indicó la igualdad de varianzas. Del mismo modo, el estadístico Durbin-Watson informó de la independencia de los residuos, ya que los valores de los tres modelos de regresión se encontraban dentro del rango recomendado (1.5-2.5) para 
considerar independientes las observaciones (Durbin y Watson 1). Por último, los valores del factor de inflación de la varianza (FIV) se encontraban por debajo de 10 y los índices de tolerancia eran mayores de 0.10 , lo que permitió descartar la existencia de colinealidad entre las variables independientes.

Los datos obtenidos con la macro de PROCESS son empleados para observar el poder predictivo de las variables y los efectos de moderación en los dos modelos propuestos. El programa realiza un análisis de regresión jerárquica y un análisis de moderación. El primero informa del poder predictivo de cada variable antecedente sobre su consecuente. El segundo aporta información sobre los efectos de la interacción entre autoeficacia y la edad, sobre satisfacción con la vida. Si el intervalo de confianza del efecto indirecto no incluye el 0 , entonces los resultados son significativos (Preacher y Hayes 177).

\section{Resultados}

Las correlaciones entre las variables extraídas de instrumentos estandarizados fueron significativas entre sí. La edad mostró también una correlación positiva por encima de 0.5 entre ambas. Los análisis descriptivos y las correlaciones bivariadas entre todas las variables de estudio se muestran en la Tabla 2.

Tabla 2. Correlación entre las variables del estudio

\begin{tabular}{|l|c|c|c|c|}
\cline { 2 - 5 } \multicolumn{1}{c|}{} & Media & dt & 1 & 2 \\
\hline Edad & 2.00 & 1.01 & & \\
\hline Satisfacción & 2.22 & 1.08 & .103 & \\
\hline Autoeficacia & 3.07 & 0.85 & -.105 & $.498^{* *}$ \\
\hline
\end{tabular}

Prueba $t$ de Student

Se analizan las diferencias de medias en las variables extraídas de instrumentos estandarizados en función de la edad. Los resultados del análisis de Scheffé de la prueba $t$ de Student pueden observarse en la Tabla 3. 
Tabla 3. Resultados de la t de Student

\begin{tabular}{|c|c|c|c|c|c|c|c|c|}
\cline { 2 - 9 } \multicolumn{1}{c|}{} & Edad & Media & Dt & F & Sig. & t & gl & $\begin{array}{c}\text { Sig. } \\
\text { (bilateral) }\end{array}$ \\
\hline \multirow{2}{*}{ Satisfacción } & $18-26$ & 2.11 & 1.06 & 0.271 & 0.604 & -0.786 & 58 & 0.435 \\
\cline { 2 - 9 } & $43-68$ & 2.33 & 1.11 & & & & & \\
\hline \multirow{2}{*}{ Autoeficacia } & $18-26$ & 3.16 & 0.84 & 0.063 & 0.803 & 0.801 & 58 & 0.426 \\
\cline { 2 - 10 } & $43-68$ & 2.98 & 0.86 & & & & & \\
\hline
\end{tabular}

No hay diferencias significativas entre medias en la satisfacción con la vida ni en la percepción de autoeficacia.

Análisis de Moderación

Posteriormente se usa la macro de PROCESS (Modelo 1) (Hayes 5) para testar el modelo de moderación de la Figura 1. Los resultados se muestran en la Tabla 4.

Tabla 4. Análisis de regresión y de moderación

\begin{tabular}{|l|c|c|c|c|c|c|c|c|c|}
\hline $\begin{array}{c}\text { Variable } \\
\text { predictora }\end{array}$ & $\begin{array}{c}\text { Variable } \\
\text { Criterio }\end{array}$ & B & $\boldsymbol{\beta}$ & R2 & F & T & df & LLCI & ULCI \\
\hline Autoeficacia & Satisfacción & .36 & .26 & .20 & 7.13 & 1.99 & $3-56$ & -.002 & .521 \\
\hline Edad & & .14 & .13 & & & 1.04 & & .122 & .387 \\
\hline $\begin{array}{l}\text { Autoeficacia } \mathrm{x} \\
\text { Edad }\end{array}$ & .42 & .30 & & & 2.29 & & .037 & .565 \\
\hline
\end{tabular}

En la tabla 4 se observa que atendiendo a los resultados obtenidos por el conjunto de supervivientes que ejercen prostitución, la autoeficacia y la edad no muestran poder predictivo sobre satisfacción con la vida. Sin embargo, la edad modera los efectos de la autoeficacia sobre satisfacción con la vida (Beta: 0.26, incremento de R2: 0.11, cambio significativo de F: 5.23, p<0.01. La interacción se puede observar en la Figura 2). Es decir, los efectos de la autoeficacia sobre la satisfacción con la vida varían en función de la edad de la persona. La autoeficacia presentó un impacto significativamente superior en el grupo mayor de 40 años con respecto al grupo menor de 26. De hecho, el análisis de la pendiente indica que en el grupo de menor edad la autoeficacia 
no predice la satisfacción con la vida $\left(\mathrm{r}^{2}=.04 ; p>.05\right)$, mientras que sí que lo hace en el grupo de mayor edad $\left(r^{2}=.37 ; p<.05\right)$.

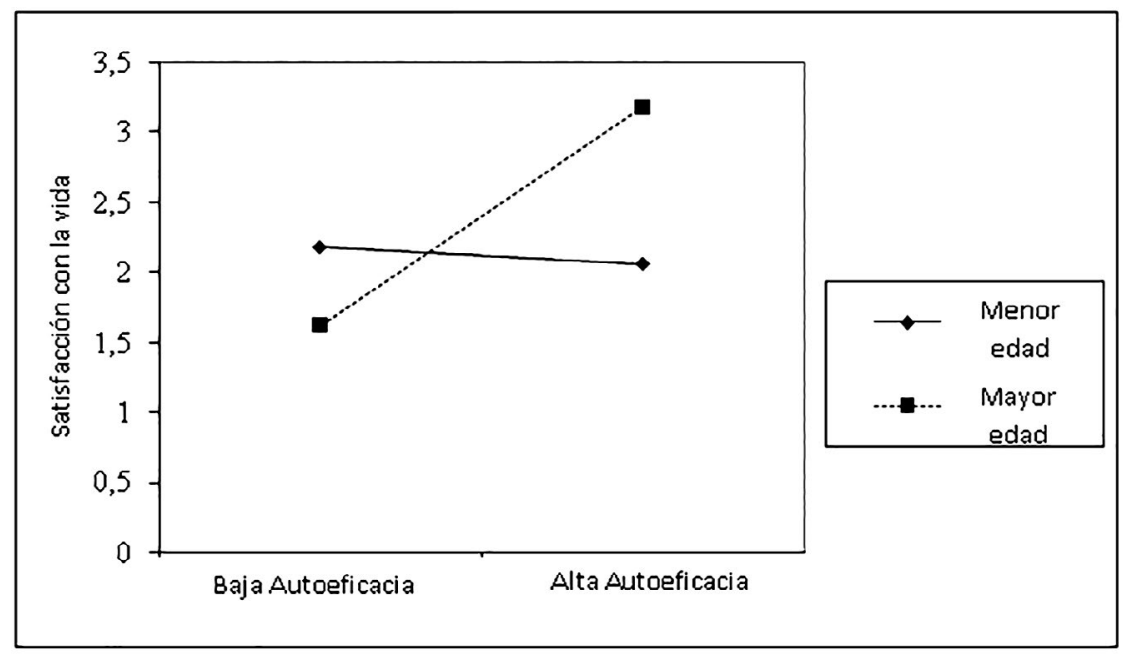

Figura 2. Resultado de la interacción

\section{Discusión}

La legislación actual contra la trata todavía necesita mejorar para asegurar la asistencia psicosocial que pretende ofrecer a quienes sobreviven a dicho crimen. Es necesario que las medidas existentes se ajusten a las situaciones y necesidades diversas que presentan las mujeres, y no que las mujeres deban ajustar su situación a las medidas ofertadas. Es decir, si existen mujeres transexuales prostitutas de 60 años que son supervivientes de trata sexual, las políticas públicas deben asegurar que las compensaciones no dependan de que sean mujeres cisgénero, realicen trabajo doméstico, y tengan 24 años. Un método efectivo para fomentar el cambio de legislación o la mejora en su operativización, es la visibilización de la existencia de colectivos desatendidos. La presente investigación, incluyendo las intereseccionalidades del estigma que supone ejercer trabajo sexual en calle siendo mayor de 40 años, ha pretendido mostrar realidades desatendidas actualmente.

Feminismo/s 31, junio 2018, pp. 39-63 
Por otra parte, la esencialización victimizante que reciben las supervivientes de trata con fines de explotación sexual, resulta perjudicial para su empoderamiento psicosocial (Frazier, Falmagne y Joffe 479). Como sostenía Brown (12), presentarlas como esencialmente vulnerables significa alejarlas de la inclusión en el imaginario social. Estamos de acuerdo con la idea de Yuval-Davisen de que la representación de víctima genuina carece de asertividad, resistencia y supervivencia, atributos reconocidos y desarrollados para liderar en países occidentales. Además, la continua vinculación entre trabajadoras sexuales y lo patológico dificulta la reducción del estigma social que deben soportar (Holgado 74). Por ello, en la presente investigación se consideró pertinente trazar el marco empoderador por medio de la conexión entre mujeres-marginalizadas-satisfechas. Es decir, mostrar que quienes encarnan el sexo débil y monstruoso, como son las mujeres supervivientes de trata que ejercen prostitución, son capaces de experimentar satisfacción con la vida, rompe con axiomas generados dentro de nuestra cultura patriarcal (Fernández Ollero 102). Esta desestabilización facilita su inclusión social y la desmitificación de la debilidad femenina.

Además, este trabajo se realiza desde el marco de la psicología positiva, la cual nos permite incrementar el bienestar mediante las fortalezas y potencialidades de las supervivientes, evitando así sobredimensionar la incidencia de alteraciones psicológicas en este sector poblacional.

Con respecto a los resultados obtenidos con el modelo que se planteó (Fig. 1), se pueden desarrollar los siguientes conocimientos:

La autoeficacia en supervivientes de trata que ejercen trabajo sexual parece mantenerse estable con independencia de su edad. Estos resultados son similares a los encontrados con población general, y son coherentes con el modelo del procesamiento dual (de Brandtstädter y Rothermund), el de selección, optimización y compensación (de Baltes y Baltes), y con la teoría motivacional del desarrollo a lo largo de la vida (de Heckhausen, Wrosch, y Schulz). Parece que, como sugieren los citados modelos, las estrategias de afrontamiento y los objetivos se van ajustando a lo largo de la vida de acuerdo a las variaciones en nuestras capacidades, por lo que las percepciones de autoeficacia se mantienen dentro de un mismo rango.

Kunzmann, Little, y Smith ya plantearon en «Is age-related stability of subjective well-being a paradox? Cross-sectional and longitudinal evidence 
from the Berlin Aging Study» la paradoja de la estabilidad a pesar de la pérdida. Esto es, aunque determinadas competencias asociadas a la eficacia mengüen, el desarrollo de otras nuevas, la modificación de los objetivos o la comparación con el grupo de iguales, producen que la percepción de autoeficacia se mantenga estable y que no se reduzca el interés por actuar de acuerdo a los propios objetivos. La percepción de límites no implica la anulación de la autoeficacia, si estos son acordes a las expectativas asociadas a la edad. Es decir, a pesar del estigma asociado al envejecimiento femenino, las mujeres que colaboraron en este estudio parece que han sido capaces de desarrollar estrategias que les han permitido sortear los daños en su percepción de eficacia. Estos resultados tienen una gran potencialidad, porque cuantifican la existencia de fortalezas en mujeres que han sido esencializadas dentro de la imagen de debilidad y patología.

Por otra parte, los resultados que se han obtenido relativos a sus niveles de satisfacción con la vida, nos indican que ésta no parece decrecer significativamente en función de la edad, si bien es cierto que tampoco experimenta el incremento que otras investigaciones señalan que se produce. No obstante, estos resultados son similares a los mostrados por otras investigaciones. Chen (516) encontró diferencias significativas moderadas y Hamarat et al. (366) obtuvieron los mismos resultados con población general. Encontrar que en supervivientes de trata con fines de explotación sexual que ejercen prostitución, la satisfacción con la vida no disminuye entre un grupo de adultas menores de 26 y otro de mayores de 42 , nos indica que algo debe suceder para que estas mujeres canalicen el estrés acumulativo asociado al envejecimiento. Desde el punto de vista psicológico parece sencillo lanzar la hipótesis de que están desarrollando fortalezas que les permiten lidiar con las violencias inherentes a crecer en los márgenes. De este modo, es posible plantearse que si existen potencialidades que les permiten amortiguar los daños, éstas puedan emplearse para desarrollar nuevas potencialidades y evitar alteraciones psicológicas futuras (Sanders y Joseph 429). Así, como Cooper y Quick (36) señalaron, tiene sentido comenzar el acompañamiento psicológico con el análisis de fortalezas, para evitar deterioros posteriores provenientes de la exposición al estrés prolongado.

Con respecto a los resultados obtenidos con el análisis de moderación, se encuentra que atendiendo a la muestra total, es decir, a los dos grupos 
de mujeres, los incrementos en autoeficacia no predicen los incrementos en satisfacción con la vida. A pesar de ello, sí que resulta significativa la interacción de autoeficacia y edad. Mientras en la población más joven parece que el sentimiento de autoeficacia no potencia la satisfacción con la vida, sí que lo hace en edades más avanzadas. Es posible que en función de la edad tengan mayor poder predictivo unas estrategias que otras en la consecución de la satisfacción con la vida. De este modo, mientras en la juventud otras competencias cumplirían el rol de la autoeficacia, en edades posteriores podría volverse central. En un sistema donde la juventud y la productividad son valores clave, la pérdida de uno puede incrementar la importancia del otro.

La observación de los resultados de la pendiente muestra que, pese a que las mujeres de mayor edad que han colaborado en esta investigación no han presentado una satisfacción con la vida significativamente mayor que las mujeres más jóvenes, si realizáramos una intervención que incrementara sus niveles de autoeficacia, lograríamos que la satisfacción con la vida de las mayores superara significativamente los niveles del grupo más joven. Sin embargo, atendiendo a los datos obtenidos en esta investigación, este mismo tipo de intervención no resultaría efectiva en el grupo más joven. Esto resulta altamente interesante dentro de un grupo poblacional como las supervivientes de trata sexual que ejercen prostitución, puesto que nos indica la necesidad de generar recursos que atiendan a su diversidad. Con el envejecimiento poblacional que se está produciendo, se han promovido notoriamente las investigaciones centradas en mejorar la calidad de vida en edades avanzadas. Sin embargo, estos avances se han centrado principalmente en lo que se denomina población general (blanca, clase media-alta...) (Fiske 791). Esta investigación aporta cifras que señalan la pertinencia de adecuar los avances a las poblaciones alejadas de la centralidad de las políticas públicas, como son las protagonistas de esta investigación.

En suma, parece que las diferentes edades implican diferentes modos de aproximarse al desarrollo del bienestar, y, por ende, diferentes niveles de satisfacción con la vida. La interacción entre autoeficacia y edad da lugar a resultados diferentes a los obtenidos por medio de su análisis independiente. En esta muestra el rol de la autoeficacia en la juventud parece tener un rol más secundario que en las edades más avanzadas (Geronimi et al. 432). 


\section{Limitaciones}

A pesar de la aplicabilidad y potencialidad de los resultados obtenidos con el modelo propuesto, la presente investigación implica varias limitaciones que deben ser tenidas en cuenta.

Se trata de una investigación pionera en el trabajo psicológico con supervivientes que realizan trabajo sexual, por ello, el análisis realizado ha sido de carácter exploratorio. Este tipo de metodología, aunque necesaria, limita el alcance de los resultados. Es conveniente realizar una investigación longitudinal para poder realizar un análisis confirmatorio. Por otra parte, sería oportuno realizar un estudio con metodología no sólo cuantitativa, también cualitativa, para poder conocer en profundidad las herramientas que emplean para lidiar con las adversidades.

El difícil acceso a población que cumpliera con las características analizadas, ha limitado también el tamaño de la muestra, y por lo tanto, el alcance de los resultados, por lo que es necesario tener en cuenta que se hayan enmascarado resultados significativos.

\section{Implicaciones prácticas}

La trata con fines de explotación sexual es violencia estructural. Las mujeres que sobreviven al crimen no tienen fácil despegarse de la violencia patriarcal que facilitó su victimización. De hecho, su continua representación como víctimas es uno de los síntomas más llamativos de ello. Con esta investigación se ha pretendido mostrar que a pesar de tener que convivir con las violencias institucionales, las supervivientes que ejercen trabajo sexual también pueden encajar dentro del ideal de bienestar promovido socialmente. A pesar de que puedan existir daños a nivel psicológico por las experiencias acumuladas, esto no supone en ningún caso la imposibilidad de experimentar satisfacción con la vida. Además, los resultados expuestos en esta investigación muestran la necesidad de eliminar el mito de la homogeneidad dentro de las personas marginalizadas. Es decir, se muestran necesidades diferentes en función de la interseccionalidad de su estigma, en este caso, con referencia a la edad. 
Mostrar esta necesidad diferencial implica, entre otros, los siguientes avances:

- Visibilización de las supervivientes de trata con fines de explotación sexual que deciden ejercer trabajo sexual.

- Inclusión de las fortalezas como autoeficacia y satisfacción con la vida, en los modelos de intervención psicológica con mujeres esencializadas en la categoría de víctimas y desviadas.

- Facilitar el proceso de inclusión social de las mujeres protagonistas de la investigación por medio del incremento en la satisfacción con la vida.

- Fortalecer el modelo psicológico extraído de la Teoría Cognitivo Social de Bandura.

- Ampliar las herramientas existentes para trabajar desde la psicología clínica feminista.

\section{REFERENCIAS BIBLIOGRÁFICAS}

Acale Sánchez, María. Derecho penal, género y nacionalidad. Granada: Ed. Comares, 2016.

Aiken, Leona S, Stephen G. West y Raymond R. Reno. Multiple regression: Testing and interpreting interactions. EEUU: Sage, 1991.

Aldama, Arturo J. (ed.). Violence and the body: race, gender, and the state. EEUU: Indiana University Press, 2003.

Arce, Constantino. Técnicas de construcción de escalas psicológicas. Madrid: Síntesis, 1994.

Armstrong, Elizabeth A., Laura Hamilton y Elizbeth M. Armstrong. «'Good Girls' Gender, Social Class, and Slut Discourse on Campus». Social Psychology Quarterly 77. 2 (2014): 100-122.

Bandura, Albert. Self-efficacy: The Exercise of Control. EEUU: Macmillan, 1997.

Bannink, Fredrike. 201 Positive Psychology Applications: Promoting Well-Being in Individuals and Communities. Nueva York: WW Norton \& Company, 2017.

Bay-Cheng, Laina y Anne E. Bruns. «Yes, but: Young women's views of unwanted sex at the intersection of gender and class». Psychology of Women Quarterly 40. 4 (2016): 504-517.

Bell, Inge Powell. «The double standard». Trans-action 8. 1-2 (1970): 75-80. 
Bernis, Cristina. «Reproducción, envejecimiento y riesgo cardiovascular en el marco del ciclo vital de las mujeres». Antropo 29 (2013): 9-19.

Brandtstädter, Jochen y Klaus Rothermund.. «The life-course dynamics of goal pursuit and goal adjustment: A two-process framework». Developmental review 22. 1 (2002): 117-150.

Brown, Wendy. States of Injury: Power and Freedom in Late Modernity. EEUU: Princeton University Press, 1995.

Brunovskis, Anette y Rebecca Surtees. «Agency or Illness. The Conceptualization of Trafficking: Victims' Choices and Behaviors in the Assistance System». Gender, Technology and Development 12. 1 (2008): 53-76.

Chen, Chaonan. «Aging and life satisfaction». Social Indicators Research 54. 1 (2001): 57-79.

Chrisler, Joan C. «Body image issues of women over 50». Women Over 50: Psychological perspectives. Eds. Varda Muhlbauer y Joan C. Chrisler, EEUU: Springer, 2007. 6-25.

Cobo, Rosa. La prostitución en el corazón del capitalismo. Madrid: La Catarata, 2017.

Connor, Kathryn M. y Jonathan R.T. Davidson. «Development of a new resilience scale: The Connor-Davidson resilience scale (CD-RISC)». Depression and anxiety 18. 2 (2003): 76-82.

Cooper, Cary L. y James Campbell Quick, eds. The Handbook of Stress and Health: A Guide to Research and Practice. EEUU: John Wiley \& Sons, 2017.

Diener, Ed et al. «The satisfaction with life scale». Journal of personality assessment 49. 1 (1985): 71-75.

Durbin, James y Geoffrey S. Watson. «Testing for serial correlation in least squares regression. III». Biometrika 58. 1 (1971): 1-19.

EU Comission. Report on the progress made in the fight against trafficking in human beings. Report from the Commission to the European Parliament and the Council, 2016.

Fernández Ollero, María Jesús. Calidad de vida y salud de las mujeres que ejercen la prostitución. Madrid: UNED, 2012.

Fiske, Susan T. «Prejudices in cultural contexts: shared stereotypes (gender, age) versus variable stereotypes (race, ethnicity, religion)». Perspectives on psychological science 12. 5 (2017): 791-799. 
Frazier, Kathryn E. y Rachel Joffe Falmagne. «Empowered victims? Women's contradictory positions in the discourse of violence prevention». Feminism E Psychology 24. 4 (2014): 479-499.

Freixas, Anne, Dolores Juliano, Isabel Holgado y Bárbara Luqu., Un sector susceptible de doble marginalización: Mujeres mayores que han ejercido la prostitución. Madrid: Instituto Universitario de Estudios de la Mujer, 2004.

Freund, Alexandra M. y Paul B. Baltes. «The orchestration of selection, optimization and compensation: An action-theoretical conceptualization of a theory of developmental regulation». Control of human behavior, mental processes, and consciousness: Essays in honor of the 60th birthday of August Flammer. Eds. Walter J. Perry y Alexander Grob. EEUU: Taylor \& Francis e-library, 2000. 35-58.

Geronimi, Elena MC; Heather L Patterson y Janet Woodruff-Borden. «Relating worry and executive functioning during childhood: the moderating role of age». Child Psychiatry \& Human Development 47.3 (2016): 430-439.

Goodwin, Robin, Olivia Cook e Yvonne Yung. «Loneliness and life satisfaction among three cultural groups». Personal Relationships 8.2 (2001): 225-230.

Hamarat, Errol, et al. «Age differences in coping resources and satisfaction with life among middle-aged, young-old, and oldest-old adults». The Journal of genetic psychology 163. 3 (2002): 360-367.

Hayes, Andrew F. Introduction to Mediation, Moderation, and Conditional Process Analysis: A Regression-based. Nueva York: Guilford Publications, 2013.

Heckhausen, Jutta, Carsten Wrosch y Richard Schulz «A motivational theory of life-span development». Psychological review 117. 1 (2010): 32.

Holgado, Isabel. Prostituciones. Diálogos sobre el sexo de pago. Barcelona: Icaria, 2009.

Jackson, Sarah Janel. «Framing Megan Williams: Intersecting discourses of race, class, and gender in television news coverage of racialized rape». Feminist Media Studies 13. 1 (2013): 46-63.

Jung, Young-Ok y Hyo-Sook Oh. «The Effects of Social Support, Self-Efficacy and Perceived Health Status on Aging Anxiety of the Middle-aged Women». Journal of Korean Public Health Nursing 30.1 (2016): 30-41.

Kunzmann, Ute, Todd D Little y Jacqui Smith. «Is age-related stability of subjective well-being a paradox? Cross-sectional and longitudinal evidence from the Berlin Aging Study». Psychology and aging 15.3 (2000): 511. 
Lai, On-Kwok. «Farewell to welfare statism! More happiness in welfare market? Putting consumption in (Post) modern context». International Journal of Social Economics 21. 1 (1994): 43-54.

Law, Tuulia. Not a sob story: Transitioning out of sex work. Canada: University of Ottawa, 2011.

Lentin, Alana y Gavan Titley. The crises of multiculturalism: Racism in a neoliberal age. Zed Books Ltd., 2011.

López Martín, Antonia, et al. Marco jurídico y análisis de la sociedad española ante la violencia de género. U de Comillas, 2017.

Losier, Gaetan F., Paul E. Bourque y Robert J Vallerand. «A motivational model of leisure participation in the elderly». The Journal of psychology 127.2 (1993): 153-170.

Lucas, Richard E. y M. Brent Donnellan. «Estimating the reliability of single-item life satisfaction measures: Results from four national panel studies». Social Indicators Research 105. 3 (2012): 323-331.

Mckenzie-Mohr, Suzanne, John Cates and Heather Mcleod. «Responding to the needs of youth who are homeless: Calling for politicized trauma-informed intervention». Children and Youth Services Review 34. 1 (2012): 136-143.

Merry, Sally Engle. The seductions of quantification: Measuring human rights, gender violence, and sex trafficking. University of Chicago Press, 2016.

Ministerio de Sanidad, Servicios Sociales e Igualdad. Plan Integral de lucha contra la trata de mujeres y niñas con fines de explotación sexual. Gobierno de España, 2015.

Pérez Alonso, Esteban. El Derecho ante las nuevas formas de esclavitud. Valencia: Tirant lo Blanch, 2017.

Perkins, Douglas D. y Marc A. Zimmerman. «Empowerment theory, research, and application». American journal of community psychology 23.5 (1995): 569-579.

Pinquart, Martin. «Correlates of subjective health in older adults: a meta-analysis». Psychology and Aging 16.3 (2001): 414-426.

Preacher, Kristopher J. y Andrew F. Hayes. «SPSS and SAS procedures for estimating indirect effects in simple mediation models». Behavior research methods 36. 4 (2004): 717-731.

Sanders, Pete y Stephen Joseph. «Person-Centered Psychology». The Wiley Handbook of Positive Clinical Psychology. Eds. Alex M. Wood y Judith Jhonson. Nueva Jersey: Wiley Blackwey, 2016. 427-444. 
Satisfacción revolucionaria. Un modelo de intervención psicológica para supervivientes de trata que hacen trabajo sexual después de los 40

Seligman, Martin. «The president's address». American Psychologist 54 (1999): 559-532.

Serrano-Parra, Maria Dolores, et al. «Validez de la escala de Resiliencia de ConnorDavidson (CD-RISC) en una población de mayores entre 60 y 75 años». International Journal of psychological research 5.2 (2012): 50-57.

Sontag, Susan. «The double standard of ageing». An ageing population. Eds Penny Liddiard y Virginia Carver. Milton Keynes: Open University Press, 1978. 629-644.

Stewart, Abigail J., Joan M. Ostrove y Ravenna Helson. «Middle aging in women: Patterns of personality change from the 30s to the 50s». Journal of Adult Development 8.1 (2001): 23-37.

Tedeschi, Richard G. y Lawrence G. Calhoun. «The Posttraumatic Growth Inventory: Measuring the positive legacy of trauma». Journal of traumatic stress 9. 3 (1996): 455-471.

Thakur, Elyse R., et al. «Medical comorbidity and distress in patients with irritable bowel syndrome: The moderating role of age». Journal of psychosomatic research 88 (2016): 48-53.

Vázquez, Carmelo, y Pau Pérez-Sales. «Emociones positivas, trauma y resistencia». Ansiedad y estrés 9. 2-3 (2003): 231-254.

Weitzer, Ronald. «Additional reflections on sex work stigma». Sexualities (2017): 1363460716684513.

Worrell, Judith. «Feminist interventions: Accountability beyond symptom reduction». Psychology of Women Quarterly 25.4 (2001): 335-343.

Yuval-Davis, Nira. Gender and Nation: SAGE Publications. Sage, 1997.

Zimmerman, Cathy, et al. «The health of trafficked women: a survey of women entering posttrafficking services in Europe». American journal of public health 98.1 (2008): 55-59.

Zimmerman, Cathy y Ligia Kiss. «Human trafficking and exploitation: A global health concern». PLoS medicine 14.11 (2017): e1002437. 